\title{
A reciprocity approach to computing generating functions for permutations with no pattern matches
}

\author{
Miles Eli Jones ${ }^{1}$ and Jeffrey Remmel ${ }^{1} \|^{\dagger}$ \\ ${ }^{1}$ Department of Mathematics, University of California, San Diego, CA, USA
}

Abstract. In this paper, we develop a new method to compute generating functions of the form

$$
N M_{\tau}(t, x, y)=\sum_{n \geq 0} \frac{t^{n}}{n !} \sum_{\sigma \in \mathcal{N M}_{n}(\tau)} x^{\operatorname{LRMin}(\sigma)} y^{1+\operatorname{des}(\sigma)}
$$

where $\tau$ is a permutation that starts with $1, \mathcal{N} \mathcal{M}_{n}(\tau)$ is the set of permutations in the symmetric group $S_{n}$ with no $\tau$-matches, and for any permutation $\sigma \in S_{n}, \operatorname{LRMin}(\sigma)$ is the number of left-to-right minima of $\sigma$ and $\operatorname{des}(\sigma)$ is the number of descents of $\sigma$. Our method does not compute $N M_{\tau}(t, x, y)$ directly, but assumes that

$$
N M_{\tau}(t, x, y)=\frac{1}{\left(U_{\tau}(t, y)\right)^{x}}
$$

where $U_{\tau}(t, y)=\sum_{n \geq 0} U_{\tau, n}(y) \frac{t^{n}}{n !}$ so that $U_{\tau}(t, y)=\frac{1}{N M_{\tau}(t, 1, y)}$. We then use the so-called homomorphism method and the combinatorial interpretation of $N M_{\tau}(t, 1, y)$ to develop recursions for the coefficient of $U_{\tau}(t, y)$.

Résumé. Dans cet article, nous développons une nouvelle méthode pour calculer les fonctions génératrices de la forme

$$
N M_{\tau}(t, x, y)=\sum_{n \geq 0} \frac{t^{n}}{n !} \sum_{\sigma \in \mathcal{N M}_{n}(\tau)} x^{\operatorname{LRMin}(\sigma)} y^{1+\operatorname{des}(\sigma)}
$$

où $\tau$ est une permutation, $\mathcal{N} \mathcal{M}_{n}(\tau)$ est l'ensemble des permutations dans le groupe symétrique $S_{n}$ sans $\tau$-matches, et pour toute permutation $\sigma \in S_{n}, \operatorname{LRMin}(\sigma)$ est le nombre de minima de gauche à droite de $\sigma$ et des $(\sigma)$ est le nombre de descentes de $\sigma$. Notre méthode ne calcule pas $N M_{\tau}(t, x, y)$ directement, mais suppose que

$$
N M_{\tau}(t, x, y)=\frac{1}{\left(U_{\tau}(t, y)\right)^{x}}
$$

où $U_{\tau}(t, y)=\sum_{n \geq 0} U_{\tau, n}(y) \frac{t^{n}}{n !}$ de sorte que $U_{\tau}(t, y)=\frac{1}{N M_{\tau}(t, 1, y)}$. Nous utilisons ensuite la méthode dite "de l'homomorphisme" et l'interprétation combinatoire de $N M_{\tau}(t, 1, y)$ pour développer des récursions sur le coefficient de $U_{\tau}(t, y)$.

Keywords: permutation, pattern match, descent, left to right minimum, symmetric polynomial, exponential generating function

\footnotetext{
${ }^{\dagger}$ Partially supported by NSF grant DMS 0654060.

1365-8050 @ 2011 Discrete Mathematics and Theoretical Computer Science (DMTCS), Nancy, France
} 


\section{Introduction}

Given a sequence $\sigma=\sigma_{1} \ldots \sigma_{n}$ of distinct integers, let $\operatorname{red}(\sigma)$ be the permutation found by replacing the $i^{\text {th }}$ largest integer that appears in $\sigma$ by $i$. For example, if $\sigma=2754$, then $\operatorname{red}(\sigma)=1432$. Given a permutation $\tau=\tau_{1} \ldots \tau_{j}$ in the symmetric group $S_{j}$, we say a permutation $\sigma=\sigma_{1} \ldots \sigma_{n} \in S_{n}$ has a $\tau$ match starting at position $i$ provided $\operatorname{red}\left(\sigma_{i} \ldots \sigma_{i+j-1}\right)=\tau$. Let $\tau-\operatorname{mch}(\sigma)$ be the number of $\tau$-matches in the permutation $\sigma$. Given a permutation $\sigma=\sigma_{1} \ldots \sigma_{n} \in S_{n}$, we let $\operatorname{des}(\sigma)=\left|\left\{i: \sigma_{i}>\sigma_{i+1}\right\}\right|$. We say that $\sigma_{j}$ is a left-to-right minimum of $\sigma$ if $\sigma_{j}<\sigma_{i}$ for all $i<j$. We let $\operatorname{LRMin}(\sigma)$ denote the number of left-to-right minima of $\sigma$.

The main goal of this paper is to give a new method to compute generating functions of the form

$$
N M_{\tau}(t, x, y)=\sum_{n \geq 0} \frac{t^{n}}{n !} N M_{\tau}(x, y)
$$

where

$$
N M_{\tau}(x, y)=\sum_{\sigma \in \mathcal{N} \mathcal{M}_{n}(\tau)} x^{\operatorname{LRMin}(\sigma)} y^{1+\operatorname{des}(\sigma)},
$$

$\tau \in S_{j}$, and $\mathcal{N} \mathcal{M}_{n}(\tau)$ is the set of permutations in $S_{n}$ with no $\tau$-matches.

Our results where motivated by results of the authors in [6] where they introduced to the study of patterns in the cycle structure of permutations. That is, suppose that $\tau=\tau_{1} \ldots \tau_{j}$ is a permutation in $S_{j}$ and $\sigma$ is a permutation in $S_{n}$ with $k$ cycles $C_{1} \ldots C_{k}$. We shall always write cycles in the form $C_{i}=\left(c_{0, i}, \ldots, c_{p_{i}-1, i}\right)$ where $c_{0, i}$ is the smallest element in $C_{i}$ and $p_{i}$ is the length of $C_{i}$ and we arrange the cycles by increasing smallest elements. That is, we arrange the cycles of $\sigma$ so that $c_{0,1}<\cdots<c_{0, k}$. Then we say that $\sigma$ has a cycle- $\tau$-match $\left(c-\tau\right.$-match) if there is an $i$ such that $C_{i}=\left(c_{0, i}, \ldots, c_{p_{i}-1, i}\right)$ where $p_{i} \geq j$ and an $r$ such that $\operatorname{red}\left(c_{r, i} c_{r+1, i} \ldots c_{r+j-1, i}\right)=\tau$ where we take indices of the form $r+s$ modulo $p_{i}$. Let $c-\tau-\operatorname{mch}(\sigma)$ be the number of cycle- $\tau$-matches in the permutation $\sigma$. For example, if $\tau=213$ and $\sigma=(1,10,9)(2,3)(4,7,5,8,6)$, then 9110 is a cycle- $\tau$-match in the first cycle and 758 and 647 are cycle- $\tau$-matches in the third cycle so that $c-\tau-\operatorname{mch}(\sigma)=3$.

Given a cycle $C=\left(c_{0}, \ldots, c_{p-1}\right)$ where $c_{0}$ is the smallest element in the cycle, we let $\operatorname{cdes}(C)=$ $1+\operatorname{des}\left(c_{0} \ldots c_{p-1}\right)$. Thus $\operatorname{cdes}(C)$ counts the number of descent pairs as we traverse once around the cycle because the extra 1 counts the descent pair $c_{p-1}>c_{0}$. For example if $C=(1,5,3,7,2)$, then cdes $(C)=3$ which counts the descent pairs 53,72 , and 21 as we traverse once around $C$. By convention, if $C=\left(c_{0}\right)$ is a one-cycle, we let $\operatorname{cdes}(C)=1$. If $\sigma$ is a permutation in $S_{n}$ with $k$ cycles $C_{1} \ldots C_{k}$, then we define $\operatorname{cdes}(\sigma)=\sum_{i=1}^{k} \operatorname{cdes}\left(C_{i}\right)$. We let $\operatorname{cyc}(\sigma)$ denote the number of cycles of $\sigma$.

In [6], Jones and Remmel studied generating functions of the form

$$
\operatorname{NCM}_{\tau}(t, x, y)=1+\sum_{n \geq 1} \frac{t^{n}}{n !} \sum_{\sigma \in \mathcal{N C M}_{n}(\tau)} x^{\operatorname{cyc}(\sigma)} y^{\operatorname{cdes}(\sigma)}
$$

where $\mathcal{N C M}_{n}(\tau)$ denote the set of permutations $\sigma \in S_{n}$ which have no cycle- $\tau$-matches. The basic approach used in that paper was to use theory of exponential structures to reduce the problem of computing $N C M_{\tau}(t, x, y)$ to the problem of computing similar generating functions for $n$-cycles. That is, let $\mathcal{N C M}_{n, k}(\tau)$ denote the set of permutations $\sigma$ of $S_{n}$ with $k$ cycles such that $\sigma$ has no cycle- $\tau$-matches and $\mathcal{L}_{m}^{n c m}(\tau)$ denote the set of $m$-cycles $\gamma$ in $S_{m}$ such $\gamma$ has no cycle- $\tau$-matches. The following theorem follows easily from the theory of exponential structures as is described in [13], for example. 


\section{Theorem 1}

$$
N C M_{\tau}(t, x, y)=1+\sum_{n \geq 1} \frac{t^{n}}{n !} \sum_{k=1}^{n} x^{k} \sum_{\sigma \in \mathcal{N C M}_{n, k}(\tau)} y^{\operatorname{cdes}(\sigma)}=e^{x \sum_{m \geq 1} \frac{t^{m}}{m !} \sum_{C \in \mathcal{L}_{m}^{n c m}(\tau)} y^{\operatorname{cdes}(C)}} .
$$

It turns out that if $\tau \in S_{j}$ is a permutation that starts with 1 , then we can reduce the problem of finding $\operatorname{NCM}_{\tau}(t, x, y)$ to the usual problem of finding the generating function of permutations that have no $\tau$-matches. That is, suppose we are given a permutation $\sigma \in S_{n}$ with $k$ cycles $C_{1} \cdots C_{k}$. Assume we have arranged the cycles so that the smallest element in each cycle is on the left and we arrange the cycles by decreasing smallest elements. Then we let $\bar{\sigma}$ be the permutation that arises from $C_{1} \cdots C_{k}$ by erasing all the parenthesis and commas. For example, if $\sigma=(7,10,9,11)(4,8,6)(1,5,3,2)$, then $\bar{\sigma}=$ 7109114861532 . It is easy to see that the minimal elements of the cycles correspond to left-to-right minima in $\bar{\sigma}$. It is also easy to see that under our bijection $\sigma \rightarrow \bar{\sigma}$, that $\operatorname{cdes}(\sigma)=\operatorname{des}(\bar{\sigma})+1$ since every left-to-right minima is part of a descent pair in $\bar{\sigma}$. For example, if $\sigma=(7,10,9,11)(4,8,6)(1,5,3,2)$ so that $\bar{\sigma}=7109114861532, \operatorname{cdes}((7,10,9,11))=2, \operatorname{cdes}((4,8,6))=2$, and $\operatorname{cdes}((1,5,3,2))=3$ so that $\operatorname{cdes}(\sigma)=2+2+3=7$ while $\operatorname{des}(\bar{\sigma})=6$. This given, Jones and Remmel [6] proved that if $\tau \in S_{j}$ and $\tau$ starts with 1 , then for any $\sigma \in S_{n}$, (1) $\sigma$ has $k$ cycles if and only if $\bar{\sigma}$ has $k$ left-to-right minima, (2) $\operatorname{cdes}(\sigma)=1+\operatorname{des}(\bar{\sigma})$, and (3) $\sigma$ has no cycle- $\tau$-matches if and only if $\bar{\sigma}$ has no $\tau$-matches. It follows that if $\tau \in S_{j}$ and $\tau$ starts with 1 , then $N C M_{\tau}(t, x, y)=N M_{\tau}(t, x, y)$. Hence, by Theorem 1. we should expect that $N M_{\tau}(t, x, y)$ is of the form $F(t, y)^{x}$ for some function $F(t, y)$. We should note that if a permutation $\tau$ does not start with 1 , then it may be that case that $\left|\mathcal{N} \mathcal{M}_{n}(\tau)\right| \neq\left|\mathcal{N C M}_{n}(\tau)\right|$. For example, Jones and Remmel [6] computed that $\left|\mathcal{N C M}_{7}(3142)\right|=4236$ and $\left|\mathcal{N} \mathcal{M}_{7}(3142)\right|=4237$.

Jones and Remmel [6] were able compute functions of the form $N C M_{\tau}(t, x, y)$ when $\tau$ starts with 1 by combinatorially proving certain recursions for the $\operatorname{NCM}_{\tau, n}(x, y)$ which led to certain sets of differential equations satisfied by $N C M_{\tau}(t, x, y)$. For example, using such methods, they were able to prove the following two theorems.

Theorem 2 Let $\tau=\tau_{1} \ldots \tau_{j} \in S_{j}$ where $j \geq 3$ and $\tau_{1}=1$ and $\tau_{j}=2$. Then

$$
N C M_{\tau}(t, x, y)=N M_{\tau}(t, x, y)=\frac{1}{\left(1-\int_{0}^{t} e^{(y-1) s-\frac{y^{\operatorname{des}(\tau)}(j-1}{(j-1) !}} d s\right)^{x}}
$$

Theorem 3 Suppose that $\tau=12 \ldots j-1 \gamma j$ where $\gamma$ is a permutation of $j+1, \ldots, j+p$ and $j \geq 3$. Then

$$
N C M_{\tau}(t, x, y)=N M_{\tau}(t, x, y)=\frac{1}{\left(U_{\tau}(t, y)\right)^{x}}
$$

where $U_{\tau}(t, y)=\sum_{n \geq 0} U_{\tau, n}(y) \frac{t^{n}}{n !}$ and for all $n \geq 0$,

$$
U_{\tau, n+j}(y)=(1-y) U_{\tau, n+j-1}(y)-y^{\operatorname{des}(\tau)}\left(\begin{array}{l}
n \\
p
\end{array}\right) U_{\tau, n-p+1}(y) .
$$

The main goal of this paper is develop a new method to obtain results similar to Theorem 3 for different classes of permutations. The basic idea of our method is not to try to compute $N C M_{\tau}(t, x, y)$ directly. 
Instead, we assume that $\tau$ starts with 1 and

$$
N M_{\tau}(t, x, y)=\left(\frac{1}{U_{\tau}(t, y)}\right)^{x} \text { where } U_{\tau}(t, y)=1+\sum_{n \geq 1} U_{\tau, n}(y) \frac{t^{n}}{n !} .
$$

Then clearly,

$$
U_{\tau}(t, y)=\frac{1}{1+\sum_{n \geq 1} N M_{\tau, n}(1, y) \frac{t^{n}}{n !}} .
$$

Remmel and various coauthors [1, 7, 8, 9, 10, 11, 12] developed a method called the homomorphism method to show that many generating functions involving permutation statistics can be derived by applying a homomorphism defined on the ring of symmetric functions $\Lambda$ to simple symmetric function identities such as

$$
H(t)=1 / E(-t)
$$

where

$$
H(t)=\sum_{n \geq 0} h_{n} t^{n}=\prod_{i \geq 1} \frac{1}{1-x_{i} t} \text { and } E(t)=\sum_{n \geq 0} e_{n} t^{n}=\prod_{i \geq 1} 1+x_{i} t
$$

are the generating functions of the homogeneous symmetric functions $h_{n}$ and the elementary symmetric functions $e_{n}$ in infinitely many variables $x_{1}, x_{2}, \ldots$ Now suppose that we define a homomorphism $\theta$ on the ring of symmetric functions $\Lambda$ in infinitely many variables $x_{1}, x_{2}, \ldots$ by setting

$$
\theta\left(e_{n}\right)=\frac{(-1)^{n}}{n !} N M_{\tau, n}(1, y)
$$

Then

$$
\theta(E(-t))=\frac{1}{\sum_{n \geq 0} N M_{\tau, n}(1, y) \frac{t^{n}}{n !}}=U_{\tau}(t, y)
$$

Thus $\theta(H(t))$ should equal $U_{\tau}(t, y)$. We shall then show how to use the combinatorial methods associated with the homomorphism method to develop recursions for the coefficient of $U_{\tau}(t, y)$ similar to those in Theorem 3 For example, we can show that

$$
\begin{aligned}
& U_{1423, n}(y)=(1-y) U_{1423, n-1}(y)+\sum_{k=1}^{\left\lfloor\frac{n-2}{2}\right\rfloor}(-y)^{k}\left(\begin{array}{c}
n-k-2 \\
k
\end{array}\right) U_{1423, n-2 k-1}(y) \text { and } \\
& U_{1324, n}(y)=(1-y) U_{1324, n-1}(y)+\sum_{k=2}^{\lfloor n / 2\rfloor}(-y)^{k-1} C_{k-1} U_{1324, n-2 k+1}(y)
\end{aligned}
$$

where $C_{k}$ is the $k$-th Catalan number.

The outline of this paper is as follows. In Section 2, we shall briefly recall the background in the theory of symmetric functions that we will need for our proofs. Then in Section 3, we shall illustrate our method by proving $(10)$ and we shall state some general results that follow from our methods. 


\section{Symmetric functions.}

In this section, we give the necessary background on symmetric functions that will be needed for our proofs.

Given a partition $\lambda=\left(\lambda_{1}, \ldots \lambda_{\ell}\right)$ where $0<\lambda_{1} \leq \ldots \leq \lambda_{\ell}$, we let $\ell(\lambda)$ be the number of nonzero integers in $\lambda$. If the sum of these integers is equal to $n$, then we say $\lambda$ is a partition of $n$ and write $\lambda \vdash n$.

Let $\Lambda$ denote the ring of symmetric functions in infinitely many variables $x_{1}, x_{2}, \ldots$. The $n^{\text {th }}$ elementary symmetric function $e_{n}=e_{n}\left(x_{1}, x_{2}, \ldots\right)$ and $n^{\text {th }}$ homogeneous symmetric function $h_{n}=$ $h_{n}\left(x_{1}, x_{2}, \ldots\right)$ are defined are defined by the generating functions given in 9$\}$. For any partition $\lambda=$ $\left(\lambda_{1}, \ldots, \lambda_{\ell}\right)$, let $e_{\lambda}=e_{\lambda_{1}} \cdots e_{\lambda_{\ell}}$ and $h_{\lambda}=h_{\lambda_{1}} \cdots h_{\lambda_{\ell}}$. It is well known that that $\left\{e_{\lambda}: \lambda\right.$ is a partition $\}$ is a basis for $\Lambda$. In particular, $e_{0}, e_{1}, \ldots$ is an algebraically independent set of generators for $\Lambda$ and, hence, a ring homomorphism $\theta$ on $\Lambda$ can be defined by simply specifying $\theta\left(e_{n}\right)$ for all $n$.

A key element of our proofs is the combinatorial description of the coefficients of the expansion of $h_{n}$ in terms of the elementary symmetric functions $e_{\lambda}$ given by Eğecioğlu and Remmel in [5]. They defined a $\lambda$-brick tabloid of shape $(n)$ to be a rectangle of height 1 and length $n$ chopped into "bricks" of lengths found in the partition $\lambda$. For example, Figure 1 shows one brick $(2,3,7)$-tabloid of shape (12).

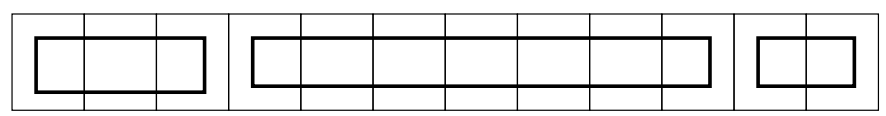

Fig. 1: A $(2,3,7)$-brick tabloid of shape (12).

Let $\mathcal{B}_{\lambda, n}$ denote the set of $\lambda$-brick tabloids of shape $(n)$ and let $B_{\lambda, n}$ be the number of $\lambda$-brick tabloids of shape $(n)$. If $B \in \mathcal{B}_{\lambda, n}$ we will write $B=\left(b_{1}, \ldots, b_{\ell(\lambda)}\right)$ if the lengths of the bricks in $B$, reading from left to right, are $b_{1}, \ldots, b_{\ell(\lambda)}$. Through simple recursions, Eğecioğlu and Remmel [5] proved that

$$
h_{n}=\sum_{\lambda \vdash n}(-1)^{n-\ell(\lambda)} B_{\lambda, n} e_{\lambda} .
$$

\section{Computing $U_{1423, n}(y)$.}

The main goal of this section is to prove the following theorem.

Theorem 4 Let $\tau=1423$. Then

$$
N M_{\tau}(t, x, y)=\left(\frac{1}{U_{\tau}(t, y)}\right)^{x} \text { where } U_{\tau}(t, y)=1+\sum_{n \geq 1} U_{\tau, n}(y) \frac{t^{n}}{n !}
$$

and $U_{\tau, 1}(y)=-y$ and for $n>1$,

$$
U_{\tau, n}(y)=(1-y) U_{\tau, n-1}(y)+\sum_{k=1}^{\left\lfloor\frac{n-2}{2}\right\rfloor}(-y)^{k}\left(\begin{array}{c}
n-(k+2) \\
k
\end{array}\right) U_{\tau, n-2 k-1}(y) .
$$


Proof: We define a ring homomorphism on the ring of symmetric functions $\Lambda$ by setting $\theta\left(e_{0}\right)=1$ and

$$
\theta\left(e_{n}\right)=\frac{(-1)^{n}}{n !} N M_{1423, n}(1, y) \text { for } n \geq 1
$$

It follows that

$$
\begin{aligned}
\theta(H(t)) & =\sum_{n \geq 0} \theta\left(h_{n}\right) t^{n}=\frac{1}{\theta(E(-t))}=\frac{1}{1+\sum_{n \geq 1}(-t)^{n} \theta\left(e_{n}\right)} \\
& =\frac{1}{1+\sum_{n \geq 1} \frac{t^{n}}{n !} N M_{1423, n}(1, y)}=1+\sum_{n \geq 1} U_{1423, n}(y) \frac{t^{n}}{n !} .
\end{aligned}
$$

Thus $\theta\left(h_{n}\right)=\frac{U_{1423, n}(y)}{n !}$. Hence, to compute $U_{1423, n}(y)$, we must compute $n ! \theta\left(h_{n}\right)$. It follows from 12 that

$$
\begin{aligned}
n ! \theta\left(h_{n}\right) & =n ! \sum_{\mu \vdash n}(-1)^{n-\ell(\mu)} B_{\mu, n} \theta\left(e_{\mu}\right) \\
& =n ! \sum_{\mu \vdash n}(-1)^{n-\ell(\mu)} \sum_{\left(b_{1}, \ldots, b_{\ell(\mu)}\right) \in \mathcal{B}_{\mu, n}} \prod_{i=1}^{\ell(\mu)} \frac{(-1)^{b_{i}}}{b_{i} !} N M_{1423, b_{i}}(1, y) \\
& =\sum_{\mu \vdash n}(-1)^{\ell(\mu)} \sum_{\left(b_{1}, \ldots, b_{\ell(\mu)}\right) \in \mathcal{B}_{\mu, n}}\left(\begin{array}{c}
n \\
b_{1}, \ldots, b_{\ell(\mu)}
\end{array}\right) \prod_{i=1}^{\ell(\mu)} N M_{1423, b_{i}}(1, y) .
\end{aligned}
$$

Our next goal is to give a combinatorial interpretation to the right-hand side of 16 . If we are given a brick tabloid $B=\left(b_{1}, \ldots, b_{\ell(\mu)}\right)$, then we can interpret the multinomial coefficient $\left(\begin{array}{c}n \\ b_{1}, \ldots, b_{\mu}\end{array}\right)$ as all ways to assign sets $S_{1}, \ldots, S_{\ell(\mu)}$ to the bricks of $B$ in such a way that $\left|S_{i}\right|=b_{i}$ for $i=1, \ldots, \ell(\mu)$ and the sets $S_{1}, \ldots, S_{\ell(\mu)}$ form a set partition of $\{1, \ldots, n\}$. Next for each brick $b_{i}$, we use the factor

$$
N M_{1423, b_{i}}(1, y)=\sum_{\sigma \in S_{b_{i}}, 1423-\operatorname{mch}(\sigma)=0} y^{\operatorname{des}(\sigma)+1}
$$

to pick a rearrangement $\sigma^{(i)}$ of $S_{i}$ which has no 1423 -matches to put in cells of $b_{i}$ and then we place a label of $y$ on each cell that starts a descent in $\sigma^{(i)}$ plus a label of $y$ on the last cell of $b_{i}$. Finally, we use the term $(-1)^{\ell(\mu)}$ to turn each label $y$ at the end of brick to a $-y$. We let $\mathcal{O}_{n}$ denote the set of all objects created in this way. For each element $O \in \mathcal{O}_{n}$, we define the weight of $O, W(O)$, to be the product of $y$ labels and the sign of $O, \operatorname{sgn}(O)$, to be $(-1)^{\ell(\mu)}$. For example, such an object $O$ constructed from the brick tabloid $B=(3,7,3)$ is pictured in Figure 2 where $W(O)=y^{7}$ and $\operatorname{sgn}(O)=(-1)^{3}$. It follows that

$$
U_{1423, n}(y)=\sum_{O \in \mathcal{O}_{n}} \operatorname{sgn}(O) W(O) .
$$

Next we define a weight-preserving sign-reversing involution $I$ on $\mathcal{O}_{n}$. Given an element $O \in \mathcal{O}_{n}$, scan the cells of $O$ from left to right looking for the first cell $c$ such that either (i) $c$ is labeled with a $y$ or (ii) $c$ is a cell at the end of a brick $b_{i}$, the element in cell $c$ is bigger than the element in the first cell of the 


\begin{tabular}{|c|c|c|c|c|c|c|c|c|c|c|c|c|}
\hline & & $-\mathbf{y}$ & & $\mathbf{y}$ & & $\mathbf{y}$ & & $\mathbf{y}$ & $-\mathbf{y}$ & $\mathbf{y}$ & & $-\mathbf{y}$ \\
\hline 4 & 7 & 11 & 8 & 10 & 5 & 12 & 3 & 9 & 6 & 2 & 1 & 13 \\
\hline
\end{tabular}

Fig. 2: An element of $\mathcal{O}_{13}$.

next brick $b_{i+1}$, and there is no 1423-match in elements of the cells in bricks $b_{i}$ and $b_{i+1}$. In case (i), if $c$ is a cell in brick $b_{j}$, then we split $b_{j}$ in to two bricks $b_{j}^{\prime}$ and $b_{j}^{\prime \prime}$ where $b_{j}^{\prime}$ contains all the cells of $b_{j}$ up to an including cell $c$ and $b_{j}^{\prime \prime}$ consists of the remaining cells of $b_{j}$ and we change to label on cell $c$ from $y$ to $-y$. In case (ii), we combine the two bricks $b_{i}$ and $b_{i+1}$ into a single brick $b$ and change the label on cell $c$ from $-y$ to $y$. For example, consider the element $O \in \mathcal{O}_{13}$ pictured in Figure 2. Even though the last element of brick 1 is bigger than the first element of brick 2, we can not combine these two bricks because the elements 711810 would be a 1423-match. Thus the first place that we can apply the involution is on cell 5 which is labeled with a $y$ so that $I(O)$ is the object pictured in Figure 3 .

\begin{tabular}{|c|c|c|c|c|c|c|c|c|c|c|c|c|}
\hline & & $-\mathbf{y}$ & & $-\mathbf{y}$ & & $\mathbf{y}$ & & $\mathbf{y}$ & $-\mathbf{y}$ & $\mathbf{y}$ & & $-\mathbf{y}$ \\
\hline 4 & 7 & 11 & 8 & 10 & 5 & 12 & 3 & 9 & 6 & 2 & 1 & 13 \\
\hline
\end{tabular}

Fig. 3: $I(O)$ for $O$ in Figure 2 .

We claim that $I$ is an involution so that $I^{2}$ is the identity. To see this, consider case (i) where we split a brick $b_{j}$ at cell $c$ which is labeled with a $y$. In that case, we let $a$ be the element in cell $c$ and $a^{\prime}$ be the element in cell $c+1$ which must also be brick $b_{j}$. It must be the case that there is no cell labeled $y$ before cell $c$ since otherwise we would not use cell $c$ to define the involution. However, we have to consider the possibility that when we spilt $b_{j}$ into $b_{j}^{\prime}$ and $b_{j}^{\prime \prime}$ that we might then be able to combine the brick $b_{j-1}$ with $b_{j}^{\prime}$ because the element in that last cell of $b_{j-1}$ is bigger that the element in the first cell of $b_{j}^{\prime}$ and there is no 1423 -match in cells of $b_{j-1}$ and $b_{j}^{\prime}$. However, the only reason that we could not combine $b_{j-1}$ and $b_{j}$ is that there must be a 1423-match in cells of $b_{j-1}$ and $b_{j}$. Clearly, this match must involve the element $a^{\prime}$. Now $a^{\prime}$ can not be the first element of a 1423-match since otherwise the 1423-match would have occurred in $b_{j}$. Thus the 1423-match in cells of $b_{j-1}$ and $b_{j}$ must have also involved $a$. Since cell $c$ was labeled with $y$, we must have $a>a^{\prime}$ which means that the only possibility is that $a$ plays the role of 4 in the 1423-match. But then, it cannot be that element in cell $c-1$ was part of brick $b_{j-1}$ because that element must play the role of 1 in the 1423-match and we are assuming that the element in that last cell of $b_{j-1}$ is bigger that the element in the first cell of $b_{j}^{\prime}$. But this would mean that there must have been a 1423-match in $b_{j}$ in the first place which contradicts the fact that there are no 1423-matches in the cells of any brick in an object $O \in \mathcal{O}_{n}$. Thus one we apply case (i), the first action that we can take is combine bricks $b_{j}^{\prime}$ and $b_{j}^{\prime \prime}$ so that $I^{2}(O)=O$.

If we are in case (ii), then again we can assume that there are no cells labeled $y$ that occur before cell $c$. When we combine brick $b_{i}$ and $b_{i+1}$, then we will label cell $c$ with a $y$. It is clear that combining the elements of $b_{i}$ and $b_{i+1}$ cannot help us combine the resulting brick $b$ with an earlier brick since it will be harder to have no 1423-matches with the larger brick $b$. Thus the first place cell $c$ where we can apply the involution will again be cell $c$ which is now labeled with a $y$ so that $I^{2}(O)=O$ if we are in case (ii). 
It is clear from our definitions that if $I(O) \neq O$, then $\operatorname{sgn}(O) W(O)=-\operatorname{sgn}(I(O)) W(I(O))$. Hence it follows from (17) that

$$
U_{1423, n}(y)=\sum_{O \in \mathcal{O}} \operatorname{sgn}(O) W(O)=\sum_{O \in \mathcal{O}, I(O)=O} \operatorname{sgn}(O) W(O) .
$$

Thus we must examine the fixed points of $I$. So assume that $O$ is a fixed point of $I$. First it is easy to see that there can be no cells which are labeled with $y$ so that elements in each brick of $O$ must be increasing. Second we cannot combine two consecutive bricks $b_{i}$ and $b_{i+1}$ in $O$ which means either that there is an increase between the bricks $b_{i}$ and $b_{i+1}$ or there is a decrease between the bricks $b_{i}$ and $b_{i+1}$ but there is a 1423-match in the cells of the bricks $b_{i}$ and $b_{i+1}$. We claim that, in addition, the elements in the first cells of the bricks must form an increasing sequence, reading from left to right. That is, suppose that $b_{i}$ and $b_{i+1}$ are two consecutive bricks in a fixed point $O$ of $I$ and that $a>a^{\prime}$ where $a$ is the element in the first cell of $b_{i}$ and $a^{\prime}$ is element in the first cell of $b_{i+1}$. Then clearly the element in the last cell of $b_{i}$ must be bigger than $a^{\prime}$ so that it must be the case that there is a 1423-match in the cells of $b_{i}$ and $b_{i+1}$. However $a^{\prime}$ is smallest element that resides in cells of of $b_{i}$ and $b_{i+1}$ which means that the only way that $a^{\prime}$ could be part of a 1423-match that occurs in the cells of $b_{i}$ and $b_{i+1}$ is to have $a^{\prime}$ play the role of 1 . But then the 1423-match would be entirely contained in $b_{i+1}$ which is impossible. Thus $a^{\prime}$ cannot be part of any 1423-match that occurs in the cells of $b_{i}$ and $b_{i+1}$. However, this would mean that the 1423-match that occurs in the cells of $b_{i}$ and $b_{i+1}$ must either be contained entirely in the cells of $b_{i}$ or entirely in the cells of $b_{i+1}$ which again is impossible. Thus it must be the case that $a<a^{\prime}$.

Thus we know that in a fixed point of $I, 1$ must be in the first cell. We claim that 2 must be either in cell 2 or 3 . That is, suppose that 2 in cell $c$ where $c \geq 4$. Then clearly cell $c$ must be the first element of its brick since otherwise the cell $c-1$ would be labeled with $y$ which is impossible for a fixed point of $I$. Now suppose that cell $c$ is part of brick $b_{i+1}$. Then the element $e$ in cell $c-1$ must be part of brick $b_{i}$. We claim that we must be able to combine bricks $b_{i}$ and $b_{i+1}$ in that case. Since 1 is in cell 1 , we know that $e>2$. Thus since $I$ is a fixed point, it must be that there is 1423-match in the cells of $b_{i}$ and $b_{i+1}$. Since $c \geq 4$, the element in cells $c-2$ and $c-1$ must both be greater than 2 . Thus the only way that 2 could be part of 1423-match in the cells of $b_{i}$ and $b_{i+1}$ if it plays the role of 1 in 1423-match. But in that case the 1423-match would occur within the cells of $b_{i+1}$ which is impossible. Thus 2 cannot be part of any 1423-match in the cells of $b_{i}$ and $b_{i+1}$ which would imply that 1423-match must occur entirely in $b_{i}$ or entirely in $b_{i+1}$. Thus it must be the case that 2 lies in cell 2 or 3 .

To summarize, we have proved the following proposition.

Proposition 5 Suppose that $O \in \mathcal{O}_{n}$ and $I(O)=O$. Then the following hold.

1. The elements within each brick of $O$ are increasing.

2. The first elements of each brick increase from left to right.

3. 1 in cell 1 and 2 in cell 2 or 3.

4. If $b_{i}$ and $b_{i+1}$ are two consecutive bricks in $O$, then either (a) there is increase between $b_{i}$ and $b_{i+1}$ or $(b)$ there is a decrease between $b_{i}$ and $b_{i+1}$ but there is 1423-match in the cells of $b_{i}$ and $b_{i+1}$.

Next we shall classify the fixed points $O \in \mathcal{O}_{n}$ of $I$. Suppose $O$ is a fixed point of I which consists of bricks $b_{1}, \ldots, b_{k}$ reading from right to left. 
Case 1. 2 is cell 2 in $O$.

In this case, there a two possibilities. Namely either (a) 1 is a brick by itself and is labeled with $-y$ or (b) 1 and 2 occupy the first two cells of the first brick. In case (a), we can remove the first brick and then we will be left with a fixed point of $I$ in $\mathcal{O}_{n-1}$ after renumbering. Thus these fixed points of type (a) will contribute $-y U_{1423, n-1}(y)$ to $U_{1423, n}(y)$. In case (b), we can collapse the first brick by removing cell 1 and again, we will be left with a fixed point of $I$ in $\mathcal{O}_{n-1}$ after renumbering. Thus fixed points of type (b) will contribute $U_{1423, n-1}(y)$ to $U_{1423, n}(y)$. Hence, the fixed points in Case 1 contribute $(1-y) U_{1423, n-1}(y)$ to $U_{1423, n}(y)$.

Case 2. 2 in cell 3 in $O$.

In this case, 2 must be the first cell of its brick since the elements are increasing in each brick. So suppose $x_{1}$ is in cell 2. Since 1 is in cell 1 , it must be the case that $x_{1}>2$. We claim that $x_{1}$ cannot be in a brick $b_{2}$ by itself since otherwise 2 starts brick $b_{3}$ and we can combine bricks $b_{2}$ and $b_{3}$. That is, in such a situation, 2 would the smallest element in the cells of bricks $b_{2}$ and $b_{3}$. Hence, the only way that 2 could be part of 1423-match in the cells of bricks $b_{2}$ and $b_{3}$ is if 2 plays the role of 1 in that match. But then the 1423-match would be entirely contained in $b_{3}$ which is impossible. Thus we would be able to combine bricks $b_{2}$ and $b_{3}$ in such a situation. It follows that $x_{1}$ must be in $b_{1}$ and there is a 1423-match in the cells of $b_{1}$ and $b_{2}$. This means that 2 can not be the only element in brick $b_{2}$. Thus it must be the case that $b_{1}$ consists of 1 and $x_{1}$ and $b_{2}$ consists of 2 followed by at least one other element.

By using the same argument that we used to show that 2 must be either in cell 2 or 3 in a fixed point of $I$, we can show that in Case 2, 3 must be either in cell 4 or 5 . Then we have the following two subcases.

Subcase 2.1. 3 is in cell 4 .

In this case, brick $b_{1}$ consists of 1 followed by $x_{1}$ and contributes a $-y$ to $\operatorname{sgn}(O) W(O)$. Then either (i) $b_{2}$ consists of the elements 2 and 3 or (ii) $b_{2}$ consists of 2 and 3 plus at least one additional element. In case (i), we can form a fixed point of $\mathcal{O}_{n-3}$ by removing brick $b_{1}$ and collapsing brick $b_{2}$ by removing 2 and renumbering. In this case, the first brick of the resulting object in $\mathcal{O}_{n-3}$ will start is brick of size 1 that contains the element 1 . In case (ii), we can also form a fixed point of $\mathcal{O}_{n-3}$ by removing brick $b_{1}$ and collapsing brick $b_{2}$ by removing 2 and renumbering. In case (ii), the first brick of the resulting object in $\mathcal{O}_{n-3}$ will start with a brick that contains 1 plus some other elements. In this way, we can get an arbitrary fixed point of $I$ in $\mathcal{O}_{n-3}$. We then have $\left(\begin{array}{c}n-3 \\ 1\end{array}\right)$ ways to pick $x_{1}$ so that the objects in Subcase 2.1 contribute $-y\left(\begin{array}{c}n-3 \\ 1\end{array}\right) U_{1423, n-3}(y)$ to $U_{1423, n}(y)$.

Subcase 2.2. 3 is in cell 5 .

Let $x_{2}$ be the element in cell 4 of $O$. Since 1 is in cell 1 and 2 in cell 3 , it follows that $x_{2}>3$ and hence 3 must start brick $b_{3}$. Thus $b_{1}$ consists of 1 and $x_{1}, b_{2}$ consists of 2 and $x_{2}$, and $1 x_{1} 2 x_{2}$ must be a 1423-match so that we must have $x_{1}>x_{2}$. There must also be a 1423-match contained in the cells of $b_{2}$ and $b_{3}$ which means that there must be at least one additional element in brick $b_{3}$.

By using the same argument that we used to show that 2 must be either in cell 2 or 3 in a fixed point of $I$, we can show that in Subcase 2.2, 4 must be either in cell 6 or 7. Then we have the following two subcases.

Subcase 2.2.1. 4 is in cell 6 . 
In this case, brick $b_{1}$ consists of 1 followed by $x_{1}$ and $b_{2}$ consists of 2 and $x_{2}$. Thus $b_{1}$ and $b_{2}$ contribute a factor of $(-y)^{2}$ to $\operatorname{sgn}(O) W(O)$. Then either (i) $b_{3}$ consists of the elements 3 and 4 or (ii) $b_{3}$ consists of 3 and 4 plus at least one additional element. In case (i), we can form a fixed point of $\mathcal{O}_{n-5}$ by removing bricks $b_{1}$, and $b_{2}$ and collapsing brick $b_{3}$ by removing 3 and renumbering. In this case, the first brick of the resulting object in $\mathcal{O}_{n-5}$ will start is brick of size 1 that contains the element 1 . In case (ii), we can also form a fixed point of $\mathcal{O}_{n-5}$ by removing bricks $b_{1}$ and $b_{2}$ and collapsing brick $b_{3}$ by removing 3 and renumbering. In case (ii), the first brick of the resulting object in $\mathcal{O}_{n-5}$ will start with a brick that contains 1 plus some other elements. In this way, we can get an arbitrary fixed point of $I$ in $\mathcal{O}_{n-5}$. We then have $\left(\begin{array}{c}n-4 \\ 2\end{array}\right)$ ways to pick $x_{1}$ and $x_{2}$ so that the objects in Subcase 2.2.1 contribute $(-y)^{2}\left(\begin{array}{c}n-4 \\ 2\end{array}\right) U_{1423, n-5}(y)$ to $U_{1423, n}(y)$.

Subcase 2.2.2. 4 is in cell 7 .

Let $x_{2}$ be the element in cell 4 of $O$ and $x_{3}$ be the element in cell 6 of $O$. Since 1 is in cell 1,2 in cell 3 , and 3 is in cell 5 , it follows that $x_{3}>4$ and hence 4 must start brick $b_{4}$. Thus $b_{1}$ consists of 1 and $x_{1}, b_{2}$ consists of 2 and $x_{2}$, and $b_{3}$ consists of 3 and $x_{3}$. Moreover, $1 x_{1} 2 x_{2}$ and $2 x_{2} 3 x_{3}$ must be 1423-matches so that we must have $x_{1}>x_{2}>x_{3}$. There must also be a 1423-match contained in the cells of $b_{3}$ and $b_{4}$ which means that there must be at least on additional element in brick $b_{4}$.

By using the same argument that we used to show that 2 must be either in cell 2 or 3 in a fixed point of $I$, we can show that in Subcase 2.2, 5 must be either in cell 8 or 9 .

Continuing this type of reasoning, one can see that the general case is where $b_{i}$ consists of $i$ and $x_{i}$ for $i=1, \ldots, k$, and brick $b_{k}$ starts with $k+1$ and has at least one additional element. Moreover, for $i=1, \ldots, k-1, i x_{i} i+1 x_{i+1}$ must be a 1423-match so that we can conclude that $x_{1}>x_{2}>\cdots>x_{k}$. Also, there a 1423-match must occur in the cells of $b_{k}$ and $b_{k+1}$ which means that there must be at least 2 elements in brick $b_{k+1}$. Then by using the same argument that we used to show that 2 must be either in cell 2 or 3 in a fixed point of $I$, we can show that in this situation, $k+2$ must be either in cell $2 k+2$ or cell $2 k+3$. We then have to consider several cases.

Case A. $n=2 k+2$.

In this case $k+2$ must be in cell $2 k+2$ which implies that $\left(x_{1}, x_{2}, \ldots, x_{k}\right)=(2 k+2,2 k+1, \ldots, k+3)$. In this case, if we remove bricks $b_{1}, \ldots, b_{k}$, remove $k+1$ from brick $b_{k+1}$, and renumber, then we obtain the configuration which consists of a single brick of size 1 which contains 1 . This is fixed point of $I$ in $\mathcal{O}_{2 k+2-(2 k+1)}=\mathcal{O}_{1}$. Note in the this case $\left\lfloor\frac{n-2}{2}\right\rfloor=k,\left(\begin{array}{c}n-k-2 \\ k\end{array}\right)=\left(\begin{array}{l}k \\ k\end{array}\right)=1$ so that the contribution to $U_{1423, n}(y)$ is

$$
(-y)^{k}\left(\begin{array}{c}
n-k-2 \\
k
\end{array}\right) U_{1423, n-2 k-1}(y)=(-y)^{k} U_{1423,1}(y)=(-y)^{k}(-y)=(-y)^{k+1}
$$

as it should be.

Case B. $n=2 k+3$.

In this case, $k+2$ cannot be in cell $2 k+3$ since otherwise brick $b_{k+1}$ must consist of $k+1$ plus another element $x_{k+1}$ and $k+2$ must be the only element in brick $b_{k+2}$. However, in that situation, $x_{k+1}>k+2$ and, hence, we could combine bricks $b_{k+1}$ and $b_{k+2}$ which would violate the fact that $O$ is a fixed point 
of $I$. Hence $k+2$ must be in cell $2 k+2$. Let $x_{k+1}$ be the element in cell $2 k+3$. It is easy to see that $x_{k}, x_{k+1}>k+2$ and that $k x_{k} k+1 k+2$ must be 1423-match. Then there are two possibilities, namely, either (a) $b_{k+1}$ contains $k+1$ and $k+2$ and $b_{k+2}$ is brick of size 1 which contains $x_{k+1}$ or (b) $b_{k+1}$ contains $k+1, k+2$, and $x_{k+1}$. In both cases, we can obtain of fixed point of $I$ in $\mathcal{O}_{2}$ by removing bricks $b_{1}, \ldots, b_{k}$, removing $k+1$ from brick $b_{k+1}$, and renumbering. In case (a), we would obtain the configuration with 2 bricks of size 1 where 1 is in the first brick and 2 is in the second brick and in case (b) we would obtain the configuration where 1 and 2 are in the same brick. These are the two configurations in $\mathcal{O}_{2}$ which are clearly fixed points of $I$. Note that we know that $x_{1}>x_{2}>\cdots x_{k}$ but we have no condition on how $x_{k+1}$ relates to $x_{1}, \ldots, x_{k}$. Thus we have $k+1$ ways to pick $x_{1}, \ldots, x_{k}$. However, note that $\left\lfloor\frac{n-2}{2}\right\rfloor=k$ and $\left(\begin{array}{c}n-(k+2) \\ k\end{array}\right)=\left(\begin{array}{c}k+1 \\ k\end{array}\right)=k+1$. Thus the contribution of the objects in Case B is equal to

$$
(-y)^{k}\left(\begin{array}{c}
n-(k+2) \\
k
\end{array}\right) U_{1423, n-(2 k+1)}=(-y)^{k}(k+1) U_{1423,2}(y)
$$

as it should be.

Case C. $n>2 k+3$ and $k+2$ is in cell $2 k+2$.

In this case, we can argue as above that if we remove bricks $b_{1}, \ldots, b_{k}$, remove $k+1$ from brick $b_{k+1}$, renumber, then we can obtain an arbitrary fixed point of $I$ in $\mathcal{O}_{n-2 k-1}$. Since we must have 1423match in the cells of $b_{k}$ and $b_{k+1}$, it must be the case that $k x_{k} k+1 k+2$ is 1423 -match so that $x_{k}>k+2$. Thus we have $\left(\begin{array}{c}n-(k+2) \\ k\end{array}\right)$ ways to pick $x_{1}, \ldots, x_{k}$. Hence, the objects in Case $\mathrm{C}$ will contribute $(-y)^{k}(\underset{k}{n-(k+2)}) U_{1423, n-2 k-1}(y)$ to $U_{1423, n}(y)$.

Case D. $n>2 k+3$ and $k+2$ is in cell $2 k+3$.

In this case, we let $x_{k+1}$ be the element in cell $2 k+2$ of $O$. Since the positions of $1, \ldots, k+2$ are accounted for in $O$, we know that $x_{k+1}>k+2$. Now $k+2$ must the be first element in brick $b_{k+2}$. Moreover, it must be the case that $k x_{k} k+1 x_{k+1}$ is 1423 -match and that there is must be a 1423-match in the cells of $b_{k+1}$ and $b_{k+2}$ so that $b_{k+2}$ must consist of at least 2 elements. Thus in Case $\mathrm{D}$, we are back in the general case that we are considering except that we are guaranteed to start with sequence of $k+1$ bricks of size 2 rather that $k$ bricks of size 2 .

Thus we have shown that $U_{1423, n}(y)$ satisfies the recursion

$$
U_{1423, n}(y)=(1-y) U_{1423, n-1}(y)+\sum_{k=1}^{\left\lfloor\frac{n-2}{2}\right\rfloor}(-y)^{k}\left(\begin{array}{c}
n-(k+2) \\
k
\end{array}\right) U_{1423, n-2 k-1}(y)
$$

with initial conditions that $U_{0}(y)=1$ and $U_{1423,1}(y)=-y$.

Similar arguments can be used to prove this type of result for equations of the form (6). For example, we can show that if $\alpha=1 q 23 \ldots(q-1)$, then $U_{\alpha, n}(y)$ satisfies the recursion

$$
U_{\alpha, n}(y)=(1-y) U_{\alpha, n-1}(y)+\sum_{k=2}^{\left\lfloor\frac{n-2}{q-2}\right\rfloor+1}(-y)^{k}\left(\begin{array}{c}
n-(k-1)(q-3)-2 \\
k-1
\end{array}\right) U_{\alpha, n-(q-2)(k-1)-1}(y)
$$


and if $\beta=12 \ldots(q-3)(q-1)(q-2) q$ where $q \geq 5$, then $U_{\beta, n}(y)$ satisfies the recursion

$$
U_{\beta, n}(y)=(1-y) U_{\beta, n-1}(y)+\sum_{k=2}^{\left\lfloor\frac{n-2}{q-2}\right\rfloor+1}(-y)^{k} U_{\beta, n-(q-2)(k-1)-1}(y) .
$$

\section{References}

[1] Desiree Beck and Jeffrey Remmel, Permutation enumeration of the symmetric group and the combinatorics of symmetric functions, J. Combin. Theory Ser. A 72 (1995), no. 1, 1-49.

[2] Francesco Brenti, Unimodal polynomials arising from symmetric functions, Proc. Amer. Math. Soc. 108 (1990), no. 4, 1133-1141.

[3] Francesco Brenti, Permutation enumeration symmetric functions, and unimodality, Pacific J. Math. 157 (1993), no. 1, 1-28.

[4] Sergi Elizalde and Marc Noy, Consecutive patterns in permutations, Adv. in Appl. Math. 30 (2003), no. 1-2, 110-125, Formal power series and algebraic combinatorics (Scottsdale, AZ, 2001).

[5] Ömer Eğecioğlu and Jeffrey Remmel, Brick tabloids and the connection matrices between bases of symmetric functions, Discrete Appl. Math. 34 (1991), no. 1-3, 107-120, Combinatorics and theoretical computer science (Washington, DC, 1989).

[6] M. Jones and J.B. Remmel, Pattern Matching in the Cycle Structures of Permutations, preprint.

[7] Thomas Langley and Jeffrey Remmel, Enumeration of m-tuples of permutations and a new class of power bases for the space of symmetric functions, Adv. Appl. Math., 36 (2006), 30-66.

[8] A. Mendes and J.B. Remmel, Generating functions for statistics on $C_{k} \prec S_{n}$, Seminaire Lotharingien de Combinatoire B54At, (2006), 40 pp.

[9] A. Mendes and J.B. Remmel, Permutations and words counted by consecutive patterns, Adv. Appl. Math, 37 4, (2006) 443-480.

[10] A. Mendes and J.B. Remmel, Descents, major indices, and inversions in permutation groups, Discrete Mathematics, Vol. 308, Issue 12, (2008), 2509-2524.

[11] A. Mendes, J.B. Remmel, and A. Riehl, Permutations with $k$-regular descent patterns, Permutation Patterns (S. Linton, N. Ruskuc, and V. Vatter, eds.), London Math. Soc. Lecture Notes 376, 259-286, (2010).

[12] J.B Remmel and A. Riehl, Generating functions for permutations which contain a given descent set, Electronic J. Combinatorics, 17 (2010), R27 33 pg.

[13] R.P. Stanley, Enumerative Combinatorics, vol. 2, Cambridge University Press, (1999).

[14] E. Steingrímsson: Generalized permutation patterns - a short survey, Permutation Patterns, St Andrews 2007, S.A. Linton, N. Ruskuc, V. Vatter (eds.), LMS Lecture Note Series, Cambridge University Press, to appear. 